\title{
Second-Look Flexible Ureterorenoscopy: A Technique Proposal to Achieve Real Stone Free Status
}

\author{
Oguz Ozden Cebeci ${ }^{1}$, Tayyar Alp Özkan², Mustafa Savas Yalcin ${ }^{3}$, Ozdal Dillioglugil ${ }^{4}$, and \\ Ibrahim Cevik ${ }^{3}$ \\ ${ }^{1}$ Saglik Bilimleri Universitesi \\ ${ }^{2}$ Acibadem Hospitals Group \\ ${ }^{3}$ Affiliation not available \\ ${ }^{4}$ Kocaeli University
}

January 22, 2021

\begin{abstract}
Introduction Residual stone fragments remain a challenging topic for urologists following retrograde intrarenal surgery (RIRS). This study investigates the effectiveness of second-look flexible ureterorenoscopy (URS) to achieve a true stone-free status and decrease stone-related events. Material and Method A total of 176 consecutive patients treated with RIRS for kidney stones were included in the study. Patients were divided into two groups, group 1 receiving the standard of care ( $\mathrm{n}=51)$ and group 2 receiving second-look flexible URS $(\mathrm{n}=125)$. In all cases, one or more calyceal stones of 1 to $4 \mathrm{~mm}$ were extracted with a basket at the time of stent removal. Unless earlier intervention was necessary, patients were followed up every six months to determine if they had experienced SREs. Results The mean follow-up time for the whole group was $21 \pm 11.1$ months. The mean number of stones in group 1 and group 2 was $1.25 \pm 0.11$ and $1.56 \pm 0.19$, respectively. Postoperative stone-free rate after RIRS for group 1 and group 2 were $37.25 \%(\mathrm{n}=19 / 51)$ and $40.8 \%(\mathrm{n}=51 / 125)$, respectively. The SFR improved to $93.6 \%(\mathrm{n}=117 / 125)$ in group 2. Multivariable analysis showed that type of intervention, stone size, and body mass index were independent prognostic factors for SREs. When group 2 was taken as a reference, the odds ratio for SREs was 8.48 (95\% CI: 2.95-24.42) in group 1. Conclusion This study found that second-look flexible URS increased the SFRs and diminished the number of SREs. We propose performing second-look flexible URS following RIRS in the presence or suspicion of RSFs to provide better treatment results, less radiation exposure, and greater patient satisfaction. Key Words Retrograde Intrarenal Surgery, Flexible Ureteroscopy, Stone-free Rate, Residual stone Fragments, Stone-Related Event, Second-look Flexible Ureteroscopy.
\end{abstract}

Second-Look Flexible Ureterorenoscopy: A Technique Proposal to Achieve Real Stone Free Status

\section{Abstract}

\section{Introduction}

Residual stone fragments remain a challenging topic for urologists following retrograde intrarenal surgery (RIRS). This study investigates the effectiveness of second-look flexible ureterorenoscopy (URS) to achieve a true stone-free status and decrease stone-related events.

\section{Material and Method}

A total of 176 consecutive patients treated with RIRS for kidney stones were included in the study. Patients were divided into two groups, group 1 receiving the standard of care $(\mathrm{n}=51)$ and group 2 receiving secondlook flexible URS $(n=125)$. In all cases, one or more calyceal stones of 1 to $4 \mathrm{~mm}$ were extracted with a 
basket at the time of stent removal. Unless earlier intervention was necessary, patients were followed up every six months to determine if they had experienced SREs.

\section{Results}

The mean follow-up time for the whole group was $21 \pm 11.1$ months. The mean number of stones in group 1 and group 2 was $1.25 \pm 0.11$ and $1.56 \pm 0.19$, respectively. Postoperative stone-free rate after RIRS for group 1 and group 2 were $37.25 \%(\mathrm{n}=19 / 51)$ and $40.8 \%(\mathrm{n}=51 / 125)$, respectively. The SFR improved to $93.6 \%$ $(\mathrm{n}=117 / 125)$ in group 2. Multivariable analysis showed that type of intervention, stone size, and body mass index were independent prognostic factors for SREs. When group 2 was taken as a reference, the odds ratio for SREs was 8.48 (95\% CI: 2.95-24.42) in group 1.

\section{Conclusion}

This study found that second-look flexible URS increased the SFRs and diminished the number of SREs. We propose performing second-look flexible URS following RIRS in the presence or suspicion of RSFs to provide better treatment results, less radiation exposure, and greater patient satisfaction.

\section{Key Words}

Retrograde Intrarenal Surgery, Flexible Ureteroscopy, Stone-free Rate, Residual stone Fragments, StoneRelated Event, Second-look Flexible Ureteroscopy.

\section{Introduction}

The most desirable conditions following a successful retrograde intrarenal surgery (RIRS) procedure are a complete flush-out of all stone fragments and diminish stone related events (SRE) ${ }^{1,2}$. One of the primary metrics used to measure the outcome of RIRS is the stone-free rate (SFR). Residual stone fragments (RSF) of $4 \mathrm{~mm}$ or less after RIRS are accepted as clinically insignificant ${ }^{3}$. RIRS studies have shown that in almost $20 \%$ of cases, clinically insignificant RSFs are observed postoperatively ${ }^{4}$; however, these RSF may grow and cause stone-related events (SRE) such as pain and infection ${ }^{5,6}$.

RSFs were detected by ultrasonography (USG), kidneys, ureters, bladder (KUB) radiography, or computed tomography $(\mathrm{CT})^{7}$. The most accurate imaging technique is the CT scan, which may show stones as small as $1 \mathrm{~mm}$, although concerns about radiation exposure limit its use ${ }^{8}$. Also, for economic reasons, CT is underutilized for imaging RSFs; therefore, a wide range of SFRs are reported following RIRS ${ }^{9-12}$.

Various techniques and methods have been reported in the literature to achieve an utterly stone-free status and to reduce radiation exposure, including artificial intelligence algorithms ${ }^{13,14}$. This study investigates the effectiveness of second-look flexible ureterorenoscopy (URS) to achieve a true stone-free status and decrease stone-related events. To the best of our knowledge, the use of routine second-look flexible URS for this purpose has not been studied previously.

\section{Patients and Methods}

We retrospectively analyzed 282 consecutive patients treated with RIRS and laser lithotripsy for kidney stones between October 2013 and December 2017. A total of 106 patients were excluded: cases in the learning curve $(n=50)$; patients who did not have double-J stent placement $(n=29)$; cases with incomplete data on the stone type, CT scan, or follow-up $(n=18)$; patients with poor visibility secondary to bleeding during RIRS $(\mathrm{n}=6)$; and cases of intra-operative complications necessitating secondary procedures $(\mathrm{n}=3)$. The remaining 176 patients comprised the study sample. They were divided into two groups based on the type of management utilized: the standard of care (group 1) group ( $\mathrm{n}=51)$, which had only an RIRS, and the second-look flexible URS (group 2) group ( $\mathrm{n}=125)$, which had a second-look flexible URS after RIRS.

We routinely performed second-look flexible URS after January 2015 to increase SFRs following a single RIRS within 2 to 3 weeks. 
All operations were performed by a single surgeon using general anesthesia, and prophylactic intravenous antibiotics were used routinely. Before placement of the ureteral access sheet, the ureter was examined with a rigid URS for the presence of ureteral stones and any other unexpected pathology. A ureteral access sheath (9.5 Fr, Flexor Cook) was used. At the end of the RIRS procedure, $4.7 \mathrm{~F}$ double-J stents were placed routinely.

RIRS was performed using a 7.5 Fr Flex X2s (Karl Storz, Germany) URS. For stone fragmentation, a Quantasystem-Litho Holmium: YAG laser (Milan, Italy) with 200-micron fiber was used. All stone fragments were extracted with a $1.7 \mathrm{~F}$ stone basket (NGage? Nitinol stone extractor, Cook, Bloomington, IN, USA) whenever possible. The second-look flexible URS procedure was performed similarly in patients who had double-J stent placement during the RIRS except for stent placement. The double-J stent was not placed after the second-look flexible URS. Extracted stone fragments were sent for X-ray diffraction stone analysis.

An immediate intraoperative SFR was defined as no stone fragments left behind and reported by the surgeon at the end of the procedure. In group 1, following RIRS and group 2 after second-look flexible URS, X-ray KUB (kidneys, ureters, and bladder), and ultrasound were performed on all patients to determine whether RSFs were present. A detectable stone of any size $(>1 \mathrm{~mm})$ was considered as a residual stone.

Unless a complication was observed, patients were discharged on postoperative day 1 . The double-J stent was removed at the second postoperative week, and a second-look flexible URS was performed at the time of stent removal. Single or multiple calyceal stones with sizes ranging from 1 to $4 \mathrm{~mm}$ were extracted with a basket. Patients with a stone size larger than $4 \mathrm{~mm}$ where laser lithotripsy needed were considered candidates for a second RIRS and thus were not included in the study. Patients who had a second-look flexible URS were discharged on the same day of the procedure.

Preoperatively, all patients had routine laboratory work and a CT scan. Unless earlier intervention was indicated, patients received follow-up for SREs every six months after that.

We analyzed the potential risk factors associated with SREs, including age, gender, body mass index (BMI), stone size, operative difficulty, CT stone density and size (centralized to the mean and scaled to $5 \mathrm{~mm}$ ), RSF, stone type, and stone management groups. SREs were defined as urinary infection, renal colic, stone growth, and any additional intervention with shockwave lithotripsy or reoperation. The operative difficulty was categorized, based on the stone location, as easy (isolated mid or upper calyx or renal pelvis stones), moderate (middle or upper calyx stones, with pelvis stones), or hard (lower calyx stone, with or without pelvis stones).

Histograms and the Shapiro-Wilk's test were used to test whether variables were normally distributed. Descriptive analyses were presented using mean+-standard deviation. The chi-square test was used to compare categories, and the t-test was used for continuous variables.

Univariable and multivariable analyses with logistic regression were used to assess the association between covariates. All analyses were performed using STATA 14.2 (StataCorp, TX). Statistical significance was set at 0.05 , and all tests were two-tailed.

\section{Results}

A total of 176 patients who had initial RIRS for kidney stones were included in this study. Age, gender, follow-up period, stone side, BMI, operative difficulty were not significantly different between groups, except for size and density (detailed demographic and clinical data of the patients are summarized in Table 1).

Immediate intraoperative SFRs for the group 1 and group 2 were $43.14 \%(\mathrm{n}=22 / 51)$ and $57.6 \%(\mathrm{n}=72 / 125)$, respectively. Postoperative radiologically controlled residual stone fragments after initial RIRS for group 1 and group 2 were $37.25 \%(\mathrm{n}=19 / 51)$ and $40.8 \%(\mathrm{n}=51 / 125)$, respectively. SFR after second-look flexible URS improved from $40.8 \%$ to $93.6 \%$ ( $\mathrm{n}=117 / 125)$; for the eight patients who were not considered stone-free, the RSFs were visibly smaller than $1 \mathrm{~mm}$ and were not retrievable with a basket. 
The univariable analysis indicated that BMI, size, operative difficulty, and type of intervention (group 1 vs. group 2) were significantly associated with SREs (Table 2). The SRE rate increased by $19.6 \%$ for each $5 \mathrm{~mm}$ increment in stone size.Age, stone density, stone type, side, gender, and radiologic RSF were not significantly associated with SREs (data not shown).

Accordingly, the final model variables were the type of intervention, operative difficulty, stone size, BMI, residual stone, and stone density $(\mathrm{p}<0.001)$. Type of intervention, stone size, and BMI were independent prognostic factors for SREs. When group 2 was taken as a reference, the odds ratio was 8.48 (95\% CI: 2.95-24.42) for SREs in group 1. The odds ratio was 1.62 (95\% CI: 1.21-2.18) for increasing stone size (Table 2).

In $69 \%(\mathrm{n}=121)$ of the whole cohort, no postoperative SRE was recorded. Urinary infection, asymptomatic stone growth, and re-operation were recorded in $10.2 \%(\mathrm{n}=18), 6.8 \%(\mathrm{n}=12)$, and $6.2 \%(\mathrm{n}=11)$ of the patients, respectively. Nine patients had postoperative SWL, four patients reported renal colic, and one patient was admitted to the emergency room due to unrelieved colic symptoms.

Renal colic ( $5.9 \%$ vs. $1 \%$ ), urinary tract infection ( $16 \%$ vs. $8 \%$ ), emergency room admission (2\% vs. 0), SWL $(15.69 \%$ vs. $0.8 \%)$, and reoperation $(13.8 \%$ vs. $3 \%)$ rates were significantly higher in the group 1 , while asymptomatic stone growth (4\% vs. $8 \%$ ) was higher in the group 2 (Table 1). Initial stone sizes were larger than $20 \mathrm{~mm}$ in patients who had reoperations in group 2.

\section{Discussion}

Surgical management of kidney stones relies mainly on the size and location of the stones. Percutaneous nephrolithotomy (PCNL) is the standard procedure for kidney stones larger than $2 \mathrm{~cm}$, and SWL or RIRS is recommended for those smaller than $2 \mathrm{~cm}^{1,2}$. However, RIRS obtains a much more common use than is approved in current urology practice ${ }^{15,16}$ because 'flexible URS is less invasive than PCNL but often with higher stone-free rates than SWL'17.

In the current study, we applied a second-look flexible URS following the RIRS procedure, regardless of stone size, thus increasing the SFRs and reducing the probability of stone related events. Definition of SFR is no stone fragments at any size except for stone size less than $1 \mathrm{~mm}$. We argue that using a second-look flexible URS in the same session with stent removal has some advantages, such as no need for additional anesthesia due to SREs, higher SFRs, and no radiation exposure for SFR confirmation.

The most desirable conditions following a successful RIRS procedure are a complete flush-out of all stone fragments and no SREs ${ }^{18}$. Great treatment results have been reported following RIRS. In the CROES study, high SFR $(85.6 \%)$ and low complication rate $(3.5 \%)$ were preserved ${ }^{9}$. Guisti et al. observed that SFR values were highest $(90.5 \%)$ in small stones at $1 \mathrm{~cm}$ but declined when the stone size increased. $(1-2 \mathrm{~cm}, 2-3 \mathrm{~cm}$ and $>3 \mathrm{~cm}$ in diameter with $78.8 \%, 70.5 \%$ and $55 \%$ respectively) ${ }^{10}$.

There are also studies reporting different SFRs when focusing on subgroups. In a review of seven RIRC studies, it was observed that SFRs ranged from $34.8 \%$ to $59.7 \%$, with Non-Contrast CT performed in the first three months postoperatively. $3.7 \%$ to $35 \%$ of these patients had to undergo stone surgery again ${ }^{11}$. Similarly, Rippel et al. reported a $38 \%$ SFR in patients who underwent CT control in the postoperative period following RIRS ${ }^{12}$.

Clinically insignificant kidney stones may not be 'insignificant', and RSFs remain a 'thorny' issue for both patients and urologists. ${ }^{13}$. Stone free status following RIRS is an independent predictor for hospital readmission and re-hospitalization ${ }^{3}$. One study reported that RSF greater than $4 \mathrm{~mm}$ would have a $59 \%$ probability of SRE, and 38\% would need reoperation. However, even if the RSF is smaller than $4 \mathrm{~mm}$, the stone's size will increase by $28 \%$ of this patient group, and $18 \%$ will need reoperation ${ }^{19}$. Complications associated with flexible URS risen from $7.7 \%$ in the perioperative period to $25.4 \%$ in the first 30 days after discharge ${ }^{20}$.

Hein et al. have studied factors influential on SREs in patients who have been followed for five years 
after RIRS. They showed that RSF of $1 \mathrm{~mm}$ or smaller after RIRS has a potential risk for SREs ${ }^{21}$. They concluded that RIRS should aim for complete stone clearance and that all RSFs should be considered significant regardless of size. In the current study, we improved our SFR and achieved lower SREs with a second-look flexible URS. Our SRE rate for the whole cohort was $31.25 \%(\mathrm{n}=55 / 176)$ at a mean follow-up of 21 months; it was higher in group $1(56.9 \%)$ than group 2 (20.8\%), a finding that supports the conclusions of Hein and colleagues.

The natural history of asymptomatic kidney stones is another controversial issue in the literature. Small, non-occlusive calyceal stones have the potential to both grow and cause pain. ${ }^{22}$. SRE is observed in more than half of asymptomatic stone patients, 5 -year average SRE observation rate is $51.2 \%$, and $14.3 \%$ had to go to the emergency department. ${ }^{23}$.

Our radiologically confirmed RSFs ( $>=1 \mathrm{~mm}$ ) after RIRS in group 2 was $59.2 \%$. Remarkably, this decreased to $6.4 \%$ after the second-look flexible URS procedure $(\mathrm{p}<0.001)$ (Table 2). Stone-related event-free patients increased significantly, from $43.14 \%$ in group 1 to $79.2 \%$ in group 2 (Table 1). Although we failed to show a significant association with RSFs in the SRE multivariable analysis, we found a significant difference between group 2 and group 1 (OR: 8.48) (Table 2). For this reason, we conclude that second-look flexible URS is beneficial because it decreases RSFs and SREs.

In our clinical practice, stent removal is performed at two weeks postoperatively. Simultaneous intervention for single or multiple stones that are retractable with a basket during stent removal provides economic and work-related advantages that may improve patient satisfaction.

Like previous studies, the current study defines SREs to include stone growth, urinary infection, an emergency room visit, or additional intervention ${ }^{6,24}$. This study found that, at a mean follow-up of 21 months, $31.25 \%$ $(\mathrm{n}=55 / 176)$ of the whole cohort were observed to have SREs, although $6.8 \%(\mathrm{n}=12 / 176)$ of those stones were asymptomatic.

Radiologically evaluated postoperative SFR after RIRS was $37.25 \%$ and $40.8 \%$ in group 1 and group 2, respectively. This difference was not statistically significant. Although we report an immediate intraoperative SFR of $57.6 \%(\mathrm{n}=72 / 125)$ for group 2, this proved to be $40.8 \%(\mathrm{n}=51 / 125)$. The difference may be due to unfavorable intraoperative conditions such as bleeding or dusting caused by low visibility. Finally, SFR increased to $93.6 \%$ after second-look flexible URS. These RSFs easy to identify when there is no dust or bleeding exists. Regarding SFR, CT scan more accurate than immediate intraoperative SFR; it carries an additional radiation exposure.

Non-Contrast CT is recommended for detecting residual stones following RIRS ${ }^{2}$ but stone patients are often at risk of exposure to excessive radiation. International Commission on Radiological Protection (ICRP) reported thresholds for safe exposure as $50 \mathrm{mSv}$ for a single year or $20 \mathrm{mSv}$ per year for five years ${ }^{25}$. Five-year retrospective radiation exposure of patients referred to a tertiary clinic for stone treatment was analyzed. Even based on CT examinations alone, it was found that $26 \%$ of these patients were exposed to more than $20 \mathrm{mSV}$ per year and $6 \%$ more than $50 \mathrm{mSV}$ per year ${ }^{26}$. It has been reported that the patients who applied to the emergency department due to acute SRE were exposed to an average of $29.7 \mathrm{mSv}$ (IQR $24.2,45.1$ ) radiation, and 20 percent of them were exposed to more than $50 \mathrm{mSv}$ in the 1-year follow-up ${ }^{27}$. We examined all patients radiologically with X-ray KUB and ultrasonography during follow-up; CT imaging was not performed of any patient.

Various techniques and methods have been reported in the literature to achieve a completely stone-free status and to reduce radiation exposure, including artificial intelligence algorithms. ${ }^{14}$. A study aimed to detect residual stone fragments with the "Endoluminal control" method. All calyceal spaces are re-controlled after lithotripsy during flexible URS; a $97 \%$ success rate has been reported compared to CT results after 4 to 8 weeks. In only one patient, they reported that a $2 \mathrm{~mm}$ residual stone fragment was missed. The authors claimed that a $\mathrm{CT}$ was not required to reduce radiation exposure when residual stone fragments were not seen after endoscopic control ${ }^{28}$. 
Danilovic et al. showed that SFR following RIRS was $93.0 \%$ accurate compared to CT when endoscopically controlled. There were no cases of RSF $>2 \mathrm{~mm}$ in CT for patients who were evaluated as stone-free on endoscopic evaluation ${ }^{29}$.

The term "second-look flexible URS" was first used by Breda et al. They used second-look flexible URS as a final diagnostic inspection after a single or repeated RIRS to confirm stone-free status. In that study, $37 \%$ $(n=19 / 51)$ of the patients had two or more RIRS procedures. Their overall SFRs after the first and second RIRS were $64.7 \%$ and $92.2 \%$, respectively. While their SFRs for stones [?]2 $\mathrm{cm}$ at first and second RIRS were $79 \%$ and $100 \%$, respectively, the SFRs for stones $>2 \mathrm{~cm}$ were $52 \%$ and $85.1 \%$, respectively ${ }^{30}$. Although they argued that the need for a second-look flexible URS would decrease with experience, our results refute this viewpoint because our group 2 had a significant decrease in SRE rates (OR: 8.48-95\%; CI: 2.95-24.42). For this reason, we believe that a routine second-look flexible URS at the time of stent removal may help reduce SREs.

Non-randomized, the retrospective design is the most important limitation of this study. Although we excluded data from the first 50 patients in the study to eliminate patients treated during the learning curve, we found that patients in group 1 were operated on relatively earlier than patients in group 2, which may be a source of bias in favor of patients in the group 2 in terms of surgical expertise. Unfortunately, we were unable to conduct a cost analysis, so further studies may help quantify the economic implications of using second-look flexible URS.

\section{Conclusion}

RSFs remain a challenging topic for urologists in the management of stone treatment. We found that secondlook flexible URS increased the SFRs and diminished the number of SREs. We also propose performing second-look flexible URS following RIRS in the presence or suspicion of RSFs to provide better treatment results, less radiation exposure, and greater patient satisfaction.

\section{References}

1. Assimos D, Krambeck A, Miller NL, et al. Surgical Management of Stones: American Urological Association/Endourological Society Guideline, PART I. J Urol. 2016;196(4):1153-1160.

2. Turk C, Petrik A, Sarica K, et al. EAU Guidelines on Interventional Treatment for Urolithiasis. Eur Urol. 2016;69(3):475-482.

3. Buldu I, Tepeler A, Karatag T, et al. Which factors affect the hospital re-admission and re-hospitalization after flexible ureterorenoscopy for kidney stone? World J Urol.2016;34(9):1291-1295.

4. Kang M, Son H, Jeong H, Cho MC, Cho SY. Clearance rates of residual stone fragments and dusts after endoscopic lithotripsy procedures using a holmium laser: 2-year follow-up results. World $J$ Urol.2016;34(11):1591-1597.

5. Candau C, Saussine C, Lang H, Roy C, Faure F, Jacqmin D. Natural history of residual renal stone fragments after ESWL. Eur Urol.2000;37(1):18-22.

6. Rebuck DA, Macejko A, Bhalani V, Ramos P, Nadler RB. The natural history of renal stone fragments following ureteroscopy. Urology.2011;77(3):564-568.

7. Acar C, Cal C. Impact of Residual Fragments following Endourological Treatments in Renal Stones. Adv Urol. $2012 ; 2012: 813523$.

8. Xiang H, Chan M, Brown V, Huo YR, Chan L, Ridley L. Systematic review and meta-analysis of the diagnostic accuracy of low-dose computed tomography of the kidneys, ureters and bladder for urolithiasis. J Med Imaging Radiat Oncol. 2017.

9. de la Rosette J, Denstedt J, Geavlete P, et al. The clinical research office of the endourological society ureteroscopy global study: indications, complications, and outcomes in 11,885 patients. J Endourol. 
2014;28(2):131-139.

10. Giusti G, Proietti S, Villa L, et al. Current Standard Technique for Modern Flexible Ureteroscopy: Tips and Tricks. Eur Urol.2016;70(1):188-194.

11. Tokas T, Habicher M, Junker D, et al. Uncovering the real outcomes of active renal stone treatment by utilizing non-contrast computer tomography: a systematic review of the current literature. World J Urol. 2017;35(6):897-905.

12. Rippel CA, Nikkel L, Lin YK, et al. Residual fragments following ureteroscopic lithotripsy: incidence and predictors on postoperative computerized tomography. J Urol. 2012;188(6):2246-2251.

13. Prezioso D, Barone B, Di Domenico D, Vitale R. Stone residual fragments: A thorny problem. Urologia. 2019;86(4):169-176.

14. Suarez-Ibarrola R, Hein S, Miernik A. Residual stone fragments: clinical implications and technological innovations. Curr Opin Urol. 2019;29(2):129-134.

15. Chung DY, Kang DH, Cho KS, et al. Comparison of stone-free rates following shock wave lithotripsy, percutaneous nephrolithotomy, and retrograde intrarenal surgery for treatment of renal stones: A systematic review and network meta-analysis. PLoS One.2019;14(2):e0211316.

16. Scotland KB, Rudnick B, Healy KA, Hubosky SG, Bagley DH. Retrograde Ureteroscopic Management of Large Renal Calculi: A Single Institutional Experience and Concise Literature Review. $J$ Endourol.2018;32(7):603-607.

17. Pearle MS. Is Ureteroscopy as Good as We Think? J Urol.2016;195(4 Pt 1):823-824.

18. Kilic O, Akand M, Van Cleynenbreugel B. Retrograde intrarenal surgery for renal stones - Part 2. Turk J Urol.2017;43(3):252-260.

19. Chew BH, Brotherhood HL, Sur RL, et al. Natural History, Complications and Re-Intervention Rates of Asymptomatic Residual Stone Fragments after Ureteroscopy: a Report from the EDGE Research Consortium. J Urol. 2016;195(4 Pt 1):982-986.

20. Wolff I, May M, Hoschke B, et al. [Differences between the inpatient complication rate after ureterorenoscopy and the 30-day outcome reported by the patient - results of the German prospective BUSTER study]. Aktuelle Urol. 2019;50(1):63-70.

21. Hein S, Miernik A, Wilhelm K, et al. Endoscopically Determined Stone Clearance Predicts Disease Recurrence Within 5 Years After Retrograde Intrarenal Surgery. J Endourol. 2016;30(6):644-649.

22. Brandt B, Ostri P, Lange P, Kvist Kristensen J. Painful caliceal calculi. The treatment of small nonobstructing caliceal calculi in patients with symptoms. Scand J Urol Nephrol. 1993;27(1):75-76.

23. Li X, Zhu W, Lam W, Yue Y, Duan H, Zeng G. Outcomes of long-term follow-up of asymptomatic renal stones and prediction of stone-related events. BJU Int. 2019;123(3):485-492.

24. Burgher A, Beman M, Holtzman JL, Monga M. Progression of nephrolithiasis: long-term outcomes with observation of asymptomatic calculi. J Endourol. 2004;18(6):534-539.

25. ICRP, 1991. 1990 Recommendations of the International Commission on Radiological Protection. Ann. ICRP 21 (1-3).

26. Tzou DT, Zetumer S, Usawachintachit M, et al. Computed Tomography Radiation Exposure Among Referred Kidney Stone Patients: Results from the Registry for Stones of the Kidney and Ureter. J Endourol.2019;33(7):619-624.

27. Ferrandino MN, Bagrodia A, Pierre SA, et al. Radiation exposure in the acute and short-term management of urolithiasis at 2 academic centers. J Urol. 2009;181(2):668-672; discussion 673. 
28. Wilhelm K, Hahn O, Schoenthaler M, et al. Stone-Free Rate after Treating Kidney Stones Exceeding $10 \mathrm{~mm}$ via Flexible Ureteroscopy: Can Endoscopic Assessment Replace Low-Dose Computed Tomography Control? Urol Int. 2019;103(3):326-330.

29. Danilovic A, Cavalanti A, Rocha BA, et al. Assessment of Residual Stone Fragments After Retrograde Intrarenal Surgery. J Endourol.2018;32(12):1108-1113.

30. Breda A, Ogunyemi O, Leppert JT, Schulam PG. Flexible ureteroscopy and laser lithotripsy for multiple unilateral intrarenal stones.Eur Urol. 2009;55(5):1190-1196.

\section{Hosted file}

Table 1.pdf available at https://authorea.com/users/388260/articles/505301-second-lookflexible-ureterorenoscopy-a-technique-proposal-to-achieve-real-stone-free-status

\section{Hosted file}

Table 2.pdf available at https://authorea.com/users/388260/articles/505301-second-lookflexible-ureterorenoscopy-a-technique-proposal-to-achieve-real-stone-free-status 